\title{
Fauna helmíntica de cães e gatos provenientes de alguns municípios do Estado de São Paulo
}

\author{
Helminthic phaune of dogs and cats of some municipalities of São Paulo State
}

\author{
Heloisa Cristina da Silva ${ }^{1,2}$; Karina Carrão Castagnolli, ${ }^{1} ;$ Daniela Miyasaka da Silveira, ${ }^{1,2}$ \\ Gustavo Henrique Nogueira Costa ${ }^{1,2}$; Ricardo Alexandre Gomes ${ }^{2}$; Adjair Antônio do Nascimento ${ }^{1}$
}

\begin{abstract}
Resumo: Foram submetidos à necropsia 28 cães e 11 gatos, provenientes dos canis municipais de Araraquara, Cosmorama, Jaboticabal, Pontal, Sertãozinho e Taiúva, Estado de São Paulo. Os animais, machos ou fêmeas, sem raça definida, de diferentes faixas etárias e naturalmente infectados por parasitos, foram alocados no "Setor de Cães e Gatos" do CPPAR - Centro de Pesquisas em Sanidade Animal - UNESP, durante cinco dias (período de adaptação), recebendo água e ração "ad libitum". O sacrifício dos animais foi efetuado de acordo com os procedimentos anestésicos recomendados pelas normas éticas utilizadas na pesquisa científica. Nos cães, 1010 helmintos foram diagnosticados. A ocorrência e amplitude de infecção foram: Toxocara canis (71,43\% e 0 - 58), Ancylostoma caninum (67,86\% e 0 - 77), Ancylostoma braziliense (57,14\% e 0 - 117), Dipylidium caninum (42,86\% e $0-73)$ e Physaloptera praeputialis ( $3,57 \%$ e 0 -1). Dos 750 helmintos diagnosticados nos felinos, a distribuição de acordo com a ocorrência e amplitude de infecção foi: A.caninum (100\% e $3-275)$, D. caninum (54,54\% e $0-33)$, Hydatigera taeniformis $(45,45 \%$ e $0-45)$, $P$. praeputialis $(54,54 \%$ e $0-20)$ e Platynosomum fastosum $(27,27 \%$ e $0-5)$.
\end{abstract}

Palavras-chaves: helmintos, cães, gatos.

Abstract: Twenty eight dogs and 11 cats from São Paulo State Municipalities (Araraquara, Cosmorama, Jaboticabal, Pontal, Sertãozinho and Taiuva cities) were submeeted to nechropsy. The animals, males and females, no defined race, with different ages and naturally infected by parasites were transported to the CPPAR - Centro de Pesquisas em Sanidade Animal - UNESP. After a five days period, in which the animals have recived water and ration "ad libitum", they were killed under normal ethic anesthetic scientific research procedures. A total of 1010 parasites were identified in the dogs, with the following occurrence and infection range: Toxocara canis (71,43\% and 0 to $58 \%$ ); Ancylostoma caninum (76,14\% and 0 to 77 ), Ancylostoma braziliense (57,14\% and 0 to 117); Dipylidium caninum (42,86\% and 0 to 73 ) and Physaloptera praeputialis $(3,57 \%$ and 0 to 1$)$. Among the 750 helminths identified in the felidae the occurrence and infection range were: A.caninum (100\% and 3 to 275$)$, D. caninum (54,54\% and 0 to 33$)$, Hydatigera taeniformis $(45,45 \%$ and 0 to 45), P. praeputialis (54,54\% and 0 to 20$)$ and Platynosomum fastosum (27,27\% and 0 to 5$)$.

Key words: helminths, dogs, cats.

\section{Introdução}

A procura de animais de companhia vem aumentando, inclusive para o emprego em terapias alternativas com crianças hospitalizadas e para estímulos táteis e visuais em pessoas especiais. O estreitamento das relações entre os animais e o homem promove uma maior exposição humana aos agentes de zoonoses, principalmente de indivíduos portadores de doenças imunossupressivas, representando riscos à saúde pública.

Ademais, além de serem considerados agentes de zoonoses, os parasitos gastrintestinais possuem um papel relevante dentre as endoparasitoses caninas e felinas, constituindo-se em um dos principais fatores que interferem no desenvolvimento do animal (SILVA et al., 1999). Alguns desses parasitos, como o Toxocara canis e o Ancylostoma braziliense, causam complicações ao homem por meio de suas larvas, provocando a patologia denominada larva "migrans" visceral e cutânea, respectivamente (CHIEFFI et al., 1976). Prociv e Croese (1996), classificaram a enterite eosinofílica em humanos como uma nova zoonose, causada por Ancylostoma caninum.

Vários estudos, realizados em diferentes regiões do Brasil, demonstram a freqüência dessas parasitoses, por meio de exames coproparasitológicos e / ou necropsia dos hospedeiros. Os resultados indicam uma ampla distribuição destes helmintos pelo país.

O parasitismo em cães foi observado na cidade de Goiânia, no Estado de Goiás, por Carneiro et al. (1973) que, ao necropsiarem cães de rua, relataram as seguintes espécies de helmintos: Ancylostoma caninum, Ancylostoma braziliense, Dipylidium caninum, Spirocerca lupi, Toxocara canis e Strongyloides stercoralis. Também na cidade de Goiânia, Campos et al. (1974) necropsia-

${ }_{1}^{1}$ CPPAR - Centro de Pesquisas em Sanidade Animal, FCAV/UNESP, Campus de Jaboticabal-SP.

${ }^{2}$ Aluno de pós-graduação do Curso de Medicina Veterinária da FCAV/UNESP. 
ram 37 gatos, encontrando A. caninum, A. braziliense, Physaloptera praeputialis, D. caninum, Toxocara cati, Pseudophyllidea, Aelurostrongylus abstrususus, Acantocephalos, Hydatigera taeniaeformis.

Lara et al. (1981), em Pelotas (RS), constataram a presença de $A$. caninum, $A$. braziliense, $D$. caninum, Dioctophyma renale, S. Iupi, T. canis e Trichuris vulpis, inclusive com parasitismo misto.

Em Viçosa, MG, Araújo et al. (1986), observaram a ocorrência de Ancylostoma spp, Dipylidium spp, Toxocara spp e Trichuris spp.

Em levantamentos mais antigos, Costa et al. (1991), na cidade de Vitória (ES), relataram a ocorrência de Ancylostoma caninum, Ancylostoma braziliense, Dipylidium caninum, Dirophylaria immitis, Phagicola arnoldi, Spirocerca lupi, Stronyloides spp, Taenia spp, Toxocara canis, Trichuris vulpis.

Na região de Londrina (PR), Guimarães Junior et al. (1996), avaliando a ocorrência de helmintos em cães, relataram os gêneros Ancylostoma spp, Dipylidium spp, Toxocara spp e Trichuris spp. Outros autores como Ogassawara (1986), Cortês et al. (1988) e Gennari, et al. (1999), na cidade de São Paulo (SP), Porto et al. (1999) em Recife (PE), também observaram a ocorrência do parasitismo em cães e gatos.

Em Porto Alegre (RS), Hoffmann et al. (1990), realizando exames coprológicos em cães errantes, detectaram Ancylostoma spp, Dipylidium caninum, Toxocara canis e Trichuris vulpis em amostras de fezes.

Farias et al. (1995) em Araçatuba (SP), por meio de técnicas de flutuação em amostras de fezes de cãese gatos determinaram a freqüência de Ancylostoma spp, Dipylidium spp, Toxocara sp e Trichuris sp, sendo a infecção múltipla mais freqüente por Ancylostoma e Toxocara.

Considerando a importância da verminose gastrintestinal em cães e gatos, o objetivo deste trabalho foi determinar a fauna helmíntica nestas espécies animais, provenientes de alguns municípios do Estado de São Paulo.

\section{Material e Métodos}

Foram selecionados 28 cães e 11 gatos, machos ou fêmeas, sem raça definida, de diferentes faixas etárias, em razoável estado nutricional e naturalmente infectados por helmintos parasitos. Os animais eram provenientes dos municípios de Araraquara, Cosmorama, Jaboticabal, Pontal, Sertãozinho e Taiúva, Estado de São Paulo.

Durante o período experimental, os animais foram mantidos em gaiolas metálicas individuais no "Setor de cães e gatos do Centro de Pesquisas em Sanidade Animal" (CPPAR/FCAVJ/UNESP) e receberam água e ração comercial "ad libitum".

Estabeleceu-se um período de adaptação de cinco dias, sendo, em seguida, realizados exames coproparasitológicos, empregando a técnica de Gordon e Whitlock (1939), para determinação do grau de infecção parasitária e classificação das espécies de helmintos de acordo com os ovos encontrados.

Posteriormente, todos os animais positivos para helmintose foram anestesiados, sacrificados e necropsiados segundo a técnica parasitológica de Jacobs et al. (1994). Para colheita de formas imaturas, eventualmente encistadas na mucosa, e de escólices de cestódeos, empregou-se o método de Mello e Campos (1974).

\section{Resultados e Discussão}

As espécies de helmintos encontradas nos cães necropsiados e as respectivas percentagens de ocorrência foram Ancylostoma caninum (67,86\%), Ancylostoma braziliense (57,14\%), Dipylidium caninum (42,86\%), Physaloptera praeputialis (3,57\%), Toxocara canis $(71,43 \%)$ (Tabela 1$)$.

Tabela 1 - Ocorrência e amplitude de infecção das espécies de helmintos gastrintestinais parasitas de cães errantes dos municípios de Araraquara, Jaboticabal, Pontal, Setãozinho e Taiuva. CPPAR/FCAVJ/UNESP.

\begin{tabular}{cccc}
\hline $\begin{array}{c}\text { Espécies } \\
\text { parasitárias }\end{array}$ & $\begin{array}{c}\mathbf{N}^{\circ} \text { de cães } \\
\text { parasitados }\end{array}$ & $\begin{array}{c}\text { Ocorrência em } \\
\text { relação ao número } \\
\text { total de cães (28) }\end{array}$ & $\begin{array}{c}\text { Amplitude de } \\
\text { infecção } \\
\mathbf{N}^{\circ} \text { de } \\
\text { helmintos }\end{array}$ \\
\hline $\begin{array}{c}\text { Ancylostoma } \\
\text { caninum }\end{array}$ & 19 & $67,86 \%$ & $0-77$ \\
$\begin{array}{c}\text { Ancylostoma } \\
\text { braziliense }\end{array}$ & 16 & $57,14 \%$ & $0-117$ \\
$\begin{array}{c}\text { Dipylidium } \\
\text { caninum }\end{array}$ & 12 & $42,86 \%$ & $0-73$ \\
$\begin{array}{c}\text { Physaloptera } \\
\text { praeputialis }\end{array}$ & 1 & $3,57 \%$ & $0-1$ \\
$\begin{array}{c}\text { Toxocara canis } \\
\text { noxpor }\end{array}$ & 20 & $71,43 \%$ & $0-58$ \\
\hline
\end{tabular}

Resultados obtidos em estudos sobre prevalência e ocorrência de helmintos gastrintestinais, realizados por meio de exames coprológicos, em outras regiões do Brasil, também são representativos para avaliação de infecções helmínticas gastrintestinais das populações caninas e podem ser comparados com os dados verificados no presente trabalho. Assim, de acordo com a literatura, as percentagens encontradas de Ancylostoma spp foram predominantes sobre as outras espécies de helmintos observadas por Lara et al. (1981); Araújo et al. (1986); Cortês et al. (1988); Hoffmann et al. (1990); Costa et al. (1991); Farias et al. (1995); Porto et al. (1999). Os índices encontrados por tais autores foram, respectivamente, 98,31\%; 49,65\%; 59,83\%; 93,28\%; $88,50 \% ; 45,2 \%$ e $92,23 \%$.

Entretanto, Beloni et al. (1993) ; Guimarães Junior et al. (1996), em Londrina (PR) e Gennari et al. (1999) em São Paulo (SP) mostraram valores inferiores $(27,30 \%$; $39,82 \%$ e $20,40 \%$, respectivamente) aos supracitados. À necropsia, Porto et al. (1999), obtiveram valores, com- 
parados com este estudo, superiores de Ancylostoma caninum (90,29\%), porém inferiores para Ancylostoma braziliense (48,54\%).

O parasito de maior ocorrência, nos cães avaliados, foi o Toxocara canis $(71,43 \%)$, cujo valor foi superior ao encontrado em cães urbanos por Porto et al., (1999), também à necropsia (2,91\%); os demais autores referiram-se apenas ao gênero Toxocara spp, baseados em exames coproparasitológicos, na seguinte seqüência: Beloni et al., (1993) 14,1\%; Hoffmann et al. (1990) 9,70\%; Guimarães Junior et al. (1996) 13,72\% e Gennari et al. (1999) 8,49\%. O alto índice encontrado no presente estudo para a espécie de Toxocara canis deve-se ao fato de que a maioria dos cães necropsiados apresentavam idade inferior a um ano, favorecendo a maior freqüência deste parasito.

Para o Dipylidium caninum, o índice observado nos cães foi de $42,86 \%$, diferindo dos obtidos em exames de fezes por Araújo et al., (1986), Hoffmann et al. (1990), Costa et al. (1991), Guimarães Junior et al. (1996), Gennari et al. (1999) e Porto et al. (1999), respectivamente: 6,09\%; 1,49\%; 6,63\%; 3,82\%; 0,28\%; 0,97\%. O último autor também determinou, por meio de necropsia, índice de $32,04 \%$, sendo este valor semelhante ao obtido neste trabalho.

Dos 28 cães necropsiados, apenas em um animal foi encontrado o parasito Physaloptera praeputialis, o que representa a ocorrência de 3,57\%.

Os dados obtidos na Tabela 2 mostram as percentagens de ocorrência dos helmintos encontrados nos gatos necropsiados, os quais foram: Ancylostoma caninum 100,0\%; Hydatigera taeniformis 45,45\%; Platynosomum fastosum 27,27\%, estando a maioria dos trematódeos encontrados atipicamente no intestino delgado; Dipylidium caninum e Physaloptera preaputialis apresentaram percentagens iguais de 54,54\%, diferindo, porém, na amplitude de infecção.

Tabela 2 - Ocorrência e amplitude de infecção das espécies de helmintos gastrintestinais parasitas de felinos errantes dos municípios de Cosmorama e Jaboticabal. CPPAR/FCAVJ/UNESP.

\begin{tabular}{llll}
\hline $\begin{array}{l}\text { Espécies } \\
\text { parasitárias }\end{array}$ & $\begin{array}{l}\mathbf{N}^{\circ} \text { de cães } \\
\text { parasitados }\end{array}$ & $\begin{array}{l}\text { Ocorrência em } \\
\text { relação ao } \\
\text { número total } \\
\text { de cães (11) }\end{array}$ & $\begin{array}{l}\text { Amplitude de } \\
\text { infecção } \\
\mathbf{N}^{\circ} \text { de helmintos }\end{array}$ \\
\hline $\begin{array}{l}\text { Ancylostoma } \\
\text { caninum }\end{array}$ & 11 & $100,00 \%$ & $3-275$ \\
$\begin{array}{l}\text { Dipylidium } \\
\text { caninum }\end{array}$ & 6 & $54,54 \%$ & $0-33$ \\
$\begin{array}{l}\text { Hydatigera } \\
\text { taeniformis }\end{array}$ & 5 & $45,45 \%$ & $0-45$ \\
$\begin{array}{l}\text { Physaloptera } \\
\text { praeputialis }\end{array}$ & 6 & $54,54 \%$ & $0-20$ \\
$\begin{array}{l}\text { Platynosomum } \\
\text { fastosum }\end{array}$ & 3 & $27,27 \%$ & $0-5$ \\
\hline
\end{tabular}

Nos gatos, o parasito mais freqüentemente observado foi o $A$. caninum (100\%), seguido pelo $D$. caninum e Physaloptera praeputialis. Esses valores diferem dos obtidos com amostras fecais na cidade de São Paulo por Ogassawara et al. (1986), onde, os nematódeos mais prevalentes foram os Ascaridae $(22,3 \%)$ e os Ancylostomidae (19,5\%). Já, Gennari et al. (1999), também em São Paulo, apresentou as seguintes prevalências: Ancylostoma spp (13,37\%), Toxocara spp (34,22\%), Dipylidium caninum (10,69\%), Physaloptera praeputialis $(4,81 \%)$ e Platynosomum concinum (1,07\%). Em Goiânia, Campos et al. (1974), encontraram prevalência menor para A. caninum (73\%), D. caninum (21,5\%), $P$. praeputialis (46\%) e H. taeniformis (3\%).

Diferentemente do que ocorre em cães, a infecção por nematódeos da família Ascaridae ocorre em felinos em todas as faixas etárias, apesar de predominar em animais com até seis meses de idade, este estudo, no entanto, não foi encontrada nos gatos nenhuma espécie desta família.

As Tabelas 3 e 4 indicam os números de animais e percentagens de parasitismo único ou em associação com outros, em cães e gatos.

O gênero Ancylostoma spp foi o mais encontrado em gatos, independente desses animais serem jovens ou adultos, reforçando as observações anteriormente realizadas por outros autores, em que este nematódeo foi mais prevalente. Isto pode ser devido a ocorrência de transmissão transplacentária e transovariana em neonatos, assim como a exposição contínua a adultos, o que favorece a transmissão por via percutânea e/ou oral. O desenvolvimento da imunidade ocorre com o aumento da faixa etária (HOSKINS et al. 1982).

Os resultados obtidos evidenciam a ocorrência natural de helmintoses nos animais de companhia, principalmente os que representam riscos à saúde humana, alertando para a importância do controle de tais parasitoses com o uso de fármacos anti-helmínticos, com combinações entre princípios ativos para ampliar o espectro de ação, além de educação sanitária.

\section{Referências Bibliográficas}

ARAÚJO, R.B.; ASSIS, C.B.; FERREIRA, P.M.; DEL CARLO, R.J. Helmintoses intestinais em cães da microregião de Viçosa-MG. Arquivo Brasileiro de Medicina Veterinária e 
Tabela 3 - Ocorrência das infecções provocadas por uma ou mais espécies de helmintos gastrintestinais em cães dos municípios de Araraquara, Jaboticabal, Pontal, Sertãozinho e Taiuva. CPPAR/FCAVJ/ UNESP.

\begin{tabular}{ccc}
\hline Tipo de infecção & $\mathbf{N}^{\circ}$ de cães parasitados & Percentual (\%) \\
\hline Única & 5 & 18,5 \\
Dupla & 10 & 37,0 \\
Tripla & 8 & 29,6 \\
Quadrupla & 4 & 14,8 \\
\hline
\end{tabular}

Tabela 4 - Ocorrência das infecções provocadas por uma ou mais espécies de helmintos gastrintestinais em felinos dos municípios de Cosmorama e Jaboticabal. CPPAR/FCAVJ/ UNESP.

\begin{tabular}{ccc}
\hline Tipo de infecção & $\mathbf{N}^{\circ}$ de gatos parasitados & Percentual (\%) \\
\hline Única & 2 & 18,18 \\
Dupla & 3 & 27,3 \\
Tripla & 3 & 27,27 \\
Quadrupla & 1 & 9,1 \\
Quintupla & 2 & 18,18 \\
\hline
\end{tabular}

Zootecnia., Belo Horizonte, v. 8, n. 32, p. 197, 1986.

BELONI, S.N.E.; PEREIRA, A.B.L.; BALARIN, M.R.S. Participação de parasitas nas gastroenterites hemorrágicas em cães. In: SEMINÁRIO BRASILEIRO DE PARASITOLOGIA VETERINÁRIA, 8., 1993,Londrina. Anais...Londrina: CBPV, 1993.

CAMPOS, D.B.; GARIBALDI, I.M.; CARNEIRO, J.R. Revista de Patologia Tropical, Goiânia, v.3/4, p. 355 - 359, 1974.

CARNEIRO, J.R.; FRIETAS J.S.; PEREIRA, E., CAMPOS, D.M.B.; VEIGA JARDIM, C. Revista de Patologia Tropical, Goiânia, v.2/4, p. 401 - 404, 1973.

CHIEFFI, P.P.; MULLER, E.E.; VIOTTI, N.M.A.; MORETTI, I.G. Estudo sobre a prevalência de enteroparasitas em cães da zona urbana do município de Londrina-PR-Brasil. Científica, São Paulo, v. 4, n.1, p. 64-67, 1976.

CORTÊS, V.A.; PAIM, G.V.; ALENCAR FILHO, R.A.. Infestação por Analostomídeos em cães e gatos apreendidos em vias públicas, São Paulo, Brasil. Revista de Saúde Pública, São Paulo, v. 22, n .4, p. 341-343, 1988.

COSTA, J.O.; LIMA, W.S.; GUIMARÃES, M.P.; LIMA, E.A.M. Freqüência de endo e ectoparasitas de cães capturados nas ruas de Vitória-ES-Brasil. Comunicação, São Paulo, p.451-452, 1991.

FARIAS, N.A., CHRISTOVÃO, M.L., STOBBE, N.S. Revista Brasileira de Parasitologia Veterinária, v. 4, São Paulo, n.1, p. $57-60,1995$.

GENNARI, S.M.; KASAI, N.; PENA, H.F.J.; CORTZ, A. Ocorrência de protozoários e helmintos em amostras de fezes de cães e gatos da cidade de São Paulo. Brazilian Journal of Veterinary Research and Animal Science, São
Paulo, v. 36, n. 2, , 1999.

GORDON, J.R.; WHITLOCK, A. V. A new technique for couting nematode eggs in sheep faeces. Journal Counsil Science Industry Research Australian, v. 12, p. 50-52, 1939.

GUIMARÃES JUNIOR, J.S.; VIDOTTO, O.; YAMAMURA, M.H.; ROSS, G.M.; FONSECA, N.A.N.; PEREIRA, A.B.L. Helmintoses gastrointestinais em cães (Canis familiaris) na região de Londrina-PR. Semina: Ci. Agr., Londrina, v. 17, n.1, p. 29-32, 1996.

HOFFMANN, R.P.; FORTES, E.; PANDOLFO, R..A.; KAISER, J.C.; BELLÓ, A.R.R.; MOTTA-NETO, A.A. Prevalência de helmintos gastrintestinais do cão errante do município de Porto Alegre, Rio Grande do Sul. Arquivo da Faculdade de Veterinária da UFRGS, Porto Alegre, v.18:, p.61-68, 1990.

HOSKINS, J.D.; MALONE, J.B.; SMITH, P.H. Prevalence of parasitism diagnosed by fecal examination in Louisiana dogs. American Journal of Veterinary Research, Chicago, v. 43, n.6, p.1106-1109, 1982.

JACOBS, D.E.; ARAKAWA, C.H.; GEMELL, M.A; McCALL, J.W.; MYERS, G.H.; VANPARIJS, O. World Association for the Advancement of Veterinary Parasitology (W.AAV.P.): Guidelines for evaluating the efficacy of anthelmintics for dogs and cats. Veterinary Parasitology, Amsterdam, v. 52, p. $179-202,1994$.

LARA S.I.M.; JAROUCO, M.RR.R.; RIBEIRO, P.B. Helmintos de Canis familiaris de Pelotas -RS. Arquivo Brasileiro de Medicina Veterinária e Zootecnia, Belo Horizonte, v.33, n. 2, p. 293-297, 1981.

MELLO, E. B. F. \& CAMPOS, M. S. Nova Técnica de coleta de helmintos parasitas intestinais. Arquivos do Instituto Biológico, São Paulo, v. 41, p. 201 - 206, 1974. 
OGASSAWARA, S. Prevalência de infecções helmínticas em gatos na cidade de São Paulo. Revista da Faculdade de Medicina Veterinária e Zootecnia da Universidade de São Paulo, São Paulo, v.23, p. 145 - 149, 1986.

PORTO, W.J.N.; ALVES, L.C.; FAUSTINO, M.A.G.; SOUTO MAIOR, M.P. Diagnóstico parasitológico de fezes $X$ diagnóstico pós - mortem em cães da cidade do Recife. In: SEMINÁRIO BRASILEIRO DE PARASITOLOGIA VET.ERINÁRIA, 11., 1999, Salvador-BA. Anais... Salvador: CBPV, 1999. p.172.

PROCIV, P.\& CROESE, J. Human enteric infection with Ancylostoma caninun: hookworms reappraised in the light of a new zoonosis. Acta - Tropica, v. 62: 1, p. 23-44, 1996.

SILVA, W.W.; JUNIOR, M.E.S.; ASSIS, L.M.; COUTO, E. A. Fauna helmíntica de cães domiciliados no sertão paraibano Patos/PB. In: SEMINÁRIO BRASILEIRO DE PARASITOLOGIA VETERINÁRIA, 11., 1999, Salvador-BA Anais... Salvador: CBPV, 1999. p.171. 